\title{
Logo Detection Based on FCM Clustering Algorithm and Texture Features
}

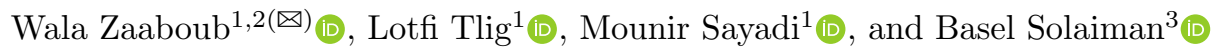 \\ 1 Research Laboratory SIME, ENSIT, Tunis, Tunisia \\ walazaaboub@gmail.com \\ 2 National Engineering School of Sfax ENIS, Sfax, Tunisia \\ 3 ITI, IMT-Atlantique, Technopole Brest, Plouzané, France
}

\begin{abstract}
Logo detection methods usually depend on logo shapes and need for training data or a-priori information on the processed images. This limits their effectiveness to real-world applications. In this paper, we tackle these challenges by exploring the textural information. Specifically we propose a novel approach for administrative logo detection based on a fuzzy classification with a multi-fractal texture feature, capable of automatically characterizing texture measures describing logo and non-logo regions. Experimental results, using two real datasets, confirm the feasibility of the proposed method for degraded administrative documents. Extensive comparative evaluations demonstrate the superiority of this approach over the state-of-the-art methods.
\end{abstract}

Keywords: Logo detection - Texture feature - Fuzzy classification • Logo extraction

\section{Introduction}

Administrative documents generally contain different salient graphical objects such as logos, signatures, seals, stamps, and bar codes. These objects are considered as a rich source of contextual information, to perfectly treat the problem of document retrieval and classification. Logo is a key visual feature for users to identify the source and the ownership of a document. Recently, many research works have been carried out on the related topics of logo retrieval, detection and recognition for images [1-3]. This paper is about the logo detection for administrative documents retrieval.

The manual identification of logos is a difficult problem, as in organization documents flow is continuously coming and growing rapidly. Consequently, many research papers focus on automatic detection and verification of logos to facilitate more reliable and appropriate documents-based systems $[4,5]$.

However, there are many challenges to have a performing and accurate document logo detection. First, administrative documents commonly contain a mixture of machine printed and handwritten text, stamps, seals, signatures, tables and other elements. Second, logo typically appears as a complex-mixture of text 
and graphic, which may lead to false detection. Third, administrative documents are generally binary images that degrade the document analysis. Fourth, logo has often a considerably disproportionate size compared to other elements of the document, it is often very small in relative to the document image resolution. The low image quality, the presence of degradation and noise in scanned documents and the large intra-class variations of logos make the detection task more difficult.

The logo detection techniques can be categorized into three categories; connected-component-based methods, local descriptor-based methods and blockbased methods. This work fall in the last category. Its main purpose is to overcome the problems of the state-of-art methods like; the high temporal complexity of the training process, the requirement of a-priori information on processed images, the difficulty of accurately processing documents with a high degree of degradation and of being unable to process correctly on unusual fonts. The proposed logo detection task will be treated as a linear three-class texture segmentation problem (logo, non-logo, and background) based on a fuzzy classifier. This paper is laid out as follows: Sect. 2 presents the proposed texture-based fuzzy approach, Sect. 3 covers the experiments and Sect. 4 concludes the work.

\section{Proposed Approach}

\subsection{Segmentation on Logo and Non-Logo Regions}

Pre-processing is a primordial phase in any analysis system that is involved in improving the image quality for further processing especially when the image is corrupted by skew, bad illumination, noise or blur. Our pre-processing phase is composed of skew correction, noise reduction and image down-scaling.

We considered that the combination of luminous intensity descriptors with texture features is a qualified process to have satisfactory image classification result in remote sensing [6]. It is on this basis that this combination will be used for administrative documents segmentation. For the texture feature, our idea is to choose the texture measures describing the different textures of administrative documents $(\log 0 /$ non-logo), and then only the features that quantify these measures are selected, instead of blindly extracting features or performing expensive tests on all possible features.

Texture features qualify measures like: density, coarseness, roughness, linearity and direction. Logo texture is often rough with an irregular structure, unlike the text zone which is in the form of repetitive patterns (alphabetic characters) spatially placed under a rule. In this case, the chosen texture measures describing the different administrative documents textures are the roughness and the regularity. In this work, multi-fractal feature is preferred because it is efficient in expressing these measures. It perfectly characterizes the administrative documents textures (logo, non-logo, and background).

We propose to adapt the multi-fractal analysis method via the wavelettransform to detect logo regions from administrative documents. Indeed, there 
are two multi-fractal-based methods: the first one generates the local information that is represented by the singularity exponent (Hölder exponent), and the second method provides the global and the local information that are given by the multi-fractal spectrum. We propose to implement the first method, as our goal is the extraction of local statistical information. What further argues our choice is that this Hölder exponent has the power to characterize the textural roughness with the advantage of multi-scale analysis [7].

The estimated singularity exponent $\alpha$ (1) is defined as follows:

$$
\alpha(\vec{x})=\lim _{r \rightarrow 0} \frac{\log T_{\psi}^{r} \mu(\vec{x})}{\log r}
$$

Where $\mu$ is the multi-fractal measure, $\psi$ is the wavelet, $\vec{x}$ is one point of the image, $r$ is an expansion factor and $T_{\psi}^{r} \mu$ is the wavelet projection.

Turiel et al. [8] defined the class of wavelet functions adapted to the singularity analysis (for which (1) is verified). For our approach, we choose the Lorentz wavelet (with the order $\gamma=1$ ) to estimate the singularity exponents. Its general expression is given by (2):

$$
\psi(\vec{x})=\left(1+|\vec{x}|^{2}\right)^{-\gamma}
$$

Using the chosen analysis method and its feature, we generate the image of Hölder exponents. The texture feature expresses a statistical moment from the generated image. We use the feature "Mean" calculated over a sliding window of the Hölder image, and assigned to the central pixel, to create the "image of texture features" that will be used in the classification step.

For luminous intensity feature, we calculate the Fisher Score [9] for every descriptor to evaluate its discrimination ability. The evaluation of this criterion on a set of test images allows the selection of the most appropriate luminous intensity descriptor characterizing the different classes (logo, non-logo, and background). Among the four statistical features ("mean", "variance", "skewness" and "kurtosis"), we choose the "mean" feature having the highest value of the characterization degree.

Using this feature expressing a statistical moment, we generate which is called the "image of intensity features". To create the "image of means" that will be used in the classification, the mean is calculated over a sliding window of the input image and assigned to the central pixel.

The Fuzzy C-Means algorithm FCM [10] is an extension of the K-Means algorithm. In fact, it presents a fuzzy concept in the calculation of the membership degree. Its goal is to build a fuzzy partition of the processed image. The image segmentation with FCM algorithm is realized using an estimation, for each pixel, of a vector of membership degrees to each class. Then, minimization of a particular objective function is required.

In this work, the FCM classification is developed in order to use a vector of features representing each pixel, instead of a pixel-based classification. The chosen features compose the vector used in the classification: the luminous intensity feature "mean" and the texture feature "Hölder exponent". This signature is 
calculated using a sliding window, with an appropriate size, centered around the corresponding pixel. The image segmentation based on FCM approach using a vector of features follows the steps presented in the following algorithm.

\section{Algorithm of FCM approach using a vector of features \\ Begin}

1. Initialization:

fuzziness parameter $m>1(m=1.1)$, threshold parameter $\varepsilon>0(\varepsilon=0.01)$, and matrix $\boldsymbol{V}^{(0)}$ of classes centers. (Size of the matrix: Number of classes $\times$ Number of features).

2. Computing the normalized matrix $\boldsymbol{F}$ of the features values. $\boldsymbol{F}$ is calculated using the original image and the image of Hölder exponents (size of $F$ : Number of pixels without edges $\times$ Number of features).

3. Computing the partition matrix $\boldsymbol{U}^{(t)}$ of elements $u_{i k}(3)$.

$$
u_{i k}=\left(\sum_{j=1}^{C}\left(\frac{\left\|F_{k}-v_{i}\right\|}{\left\|F_{k}-v_{j}\right\|}\right)^{\frac{2}{m-1}}\right)^{-1}
$$

$C$ : Number of classes, $\boldsymbol{v}_{i}$ : Vector of the center of class $i$, and $\boldsymbol{F}_{k}$ : Extracted feature vector of the $\mathrm{k}$-th pixel.

4. Updating the matrix $\boldsymbol{V}^{(t)}$ of classes centers $v_{i}(4)$.

$$
v_{i}=\frac{\sum_{k=1}^{N}\left(u_{i k}\right)^{m} F_{k}}{\sum_{k=1}^{N}\left(u_{i k}\right)^{m}}
$$

5. Test if the stop condition (5) is reached, else return to step 3.

$$
\left\|V^{(t)}-V^{(t-1)}\right\|>\varepsilon
$$

$\boldsymbol{V}^{(t)}$ : matrix of class centers calculated in the current iteration, and $\boldsymbol{V}^{(t-1)}$ : matrix of class centers calculated in the previous iteration.

\section{End}

The main advantage of the algorithm comes from the use of membership degrees. Due to them, the iterative optimization process become more robust, by taking into account overlaps between classes. Thus, it allows to obtain partitions that are more relevant and closer to reality. In addition, these degrees allow to take nuanced decisions for the pixel assignation to a class, which is very interesting for any classification type. Among the other advantages of the algorithm, we can note that its complexity is relatively reduced compared to other non-supervised classification, this makes it exploitable to deal with large problems (big amount of data). This strong point argues our classifier choice for administrative documents, as in organization documents flow is continuously coming and growing rapidly and usually they are high resolution documents. A summarizing schema of the proposed segmentation (logo, non-logo, and background) for administrative documents is shown in Fig. 1. 
One of the fundamental issues of implementing classification algorithm is that the system cannot have error-free information about the interest region. In addition, for logos contained near-scattered components, an ordinary extraction algorithm is typically unable to detect all these components. Therefore, as a postsegmentation, the proposed system deployed the morphological operations [11] for a better approach of refining logo region extraction. The idea is to implement both opening and closing operations in order to join separated logo sub-areas and remove the unwanted non-logo regions, which can be sometimes confused with the logo regions. One of the advantages of these two operations is that keep general shape but smooth with respect to object (for opening) or background (for closing).

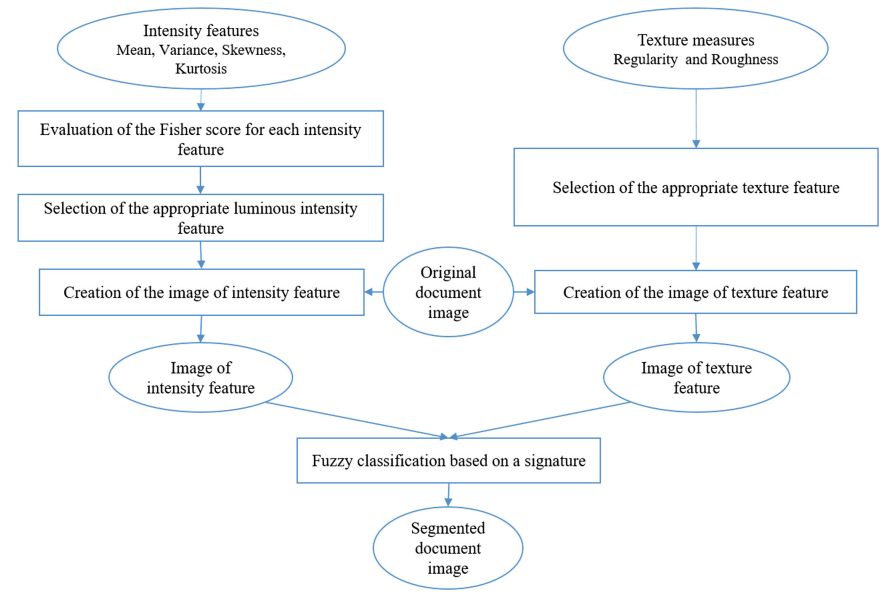

Fig. 1. Proposed schema of the segmentation of documents on: logo, non-logo, and background

\subsection{Logo Detection and Extraction}

First, the logo is located on the scanned document using bounding box surrounding this zone. Second, it is extracted from the input image taking into consideration the corresponding position of the detected zone in the segmented image. Logo localization is based on the segmented image and specific contextual rules defining shape and location of logos in administrative documents:

- Shape: The logo is typically a small compact region in relative to the document resolution.

- Location: The representational property of the logo requires placing it in very distinct locations on the document, in an isolated region not embedded in the text zone as other graphic components, generally at the document border. 
We should say that these contextual rules differ from the a-priori information that depends on the processed documents. These rules are generic and valid for most administrative documents.

\section{Experimental Study}

In this paper, two datasets are involved to evaluate the performance and effectiveness of the proposed approach. A public document image collection with realistic scope and complexity has a significant effect on the document analysis. We should note that an evaluation using large public datasets is more realistic than that using self-collected datasets which are limited and capture fewer variations.

First, experimental study is realized on the Tobacco-800 dataset [12]; a public subset of the complex document image processing test collection constructed by Illinois Institute of Technology, assembled from 42 million pages of documents (in seven million multi-page TIFF images) released by tobacco companies under the master settlement agreement and originally hosted at University of California. It is a realistic database composed of 1290 documents images collected and scanned in binary format using a wide variety of equipment over time, for document analysis and retrieval. The resolutions of these documents vary significantly from 150 to 300 DPI and the dimensions of images range from 1200 by 1600 to 2500 . Tobacco- 800 has become the standard public dataset for research works on logos in scanned documents. Ground truth labels of the logos are created using XML documents and only consist on rectangular bounding boxes on logos. In this paper, the experimentation is performed using 432 logos across 35 classes detected from this dataset. Noting that this dataset is commonly used for its significant noise, as result of the binarization and the noise of scanning devices.

Second, experiments are realized on a color document image dataset called Scanned Pseudo-Official Data-Set (SPODS) [13] containing 1080 real world official documents characterized by the presence of logos, signatures, stamps and printed text. It consists of 32 logos, 32 stamps, 32 signatures, and 12 document types. The resolution of these documents is 300 DPI. Groundtruth of this public dataset is available by annotating the different elements in documents.

A close inspection of the experimentation indicates that the proposed system delivers good results on public data and detects almost all the logos from the processed datasets. Examples of correctly detected logos from Tobacco-800 dataset and SPODS are shown in Fig. 2 and Fig. 3 respectively. Here, we can notice the robustness of multi-fractal feature and its ability to find different logos.

In previous works like in [14], the noise was detected as part of the logo. In addition, small logos degraded the recall of the detection. Unlike these works, our system has the power to detect noisy and small logos (at bottom of Fig. 2). Thus, one of the strong points of the proposed approach is that the logo detection is not affected by low image quality with much noise.

The experiments carried out on another type of documents having an adhesion of the logo and the text that do not belong to the logo area in ground truth. 


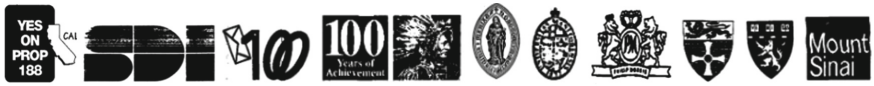
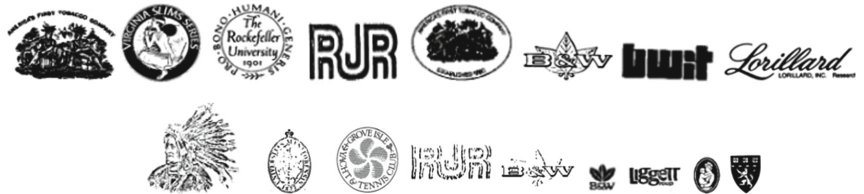

Fig. 2. Examples of correctly detected logos from the Tobacco-800 dataset

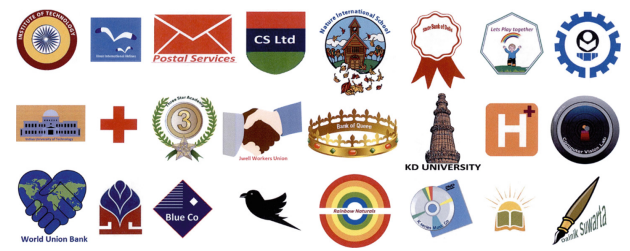

Fig. 3. Examples of correctly detected logos from the SPODS dataset

The difficulty of logo detection increases in the case where the gap between logo and text is narrow. We performed our method to show the impact of this adhesion on the logo detection. Unlike the other methods, our system managed to successfully extract logos from Tobacco-800 dataset, as depicted in Fig. 4. Thus, second strong point of our method is that it can deal with logos having close text zones. The experimentation on various scanned documents show the effective ability of the texture-based fuzzy system to locate logos.

The experiment results on SPODS dataset show that the performance of the proposed approach to detect logos is satisfactory. Another strong point of our method is that it can distinguish between logo and stamp having almost the same shape characteristics, as reported in Fig. 5. Thus, the qualitative analysis emphasizes the great results of detection process.

The quantitative analysis of images shows a visible improvement. The results indicate that the proposed system performs superbly in accuracy, precision, recall, and F-measure. By performing our system on the Tobacco- 800 dataset, we gain $98.93 \%$ on precision, $97.89 \%$ on recall and $96.86 \%$ on accuracy in document logo detection, with high F-measure value (98.41\%). The proposed approach is also successfully tested on SPODS. These results demonstrate the success of the method and highlight the importance of using a multi-fractal texture feature to correctly detect logos.

An effective comparative analysis is that where the environment of experimentation is the same for all the methods. In such case, the comparison of the performance of these methods is meaningful. In this context, we focused on the most recent and relevant methods, and only which are performed on the same Tobacco-800 dataset for automatic logo detection. The proposed logo detection yield the best result as compared to the other works, as illustrated in Table 1. 


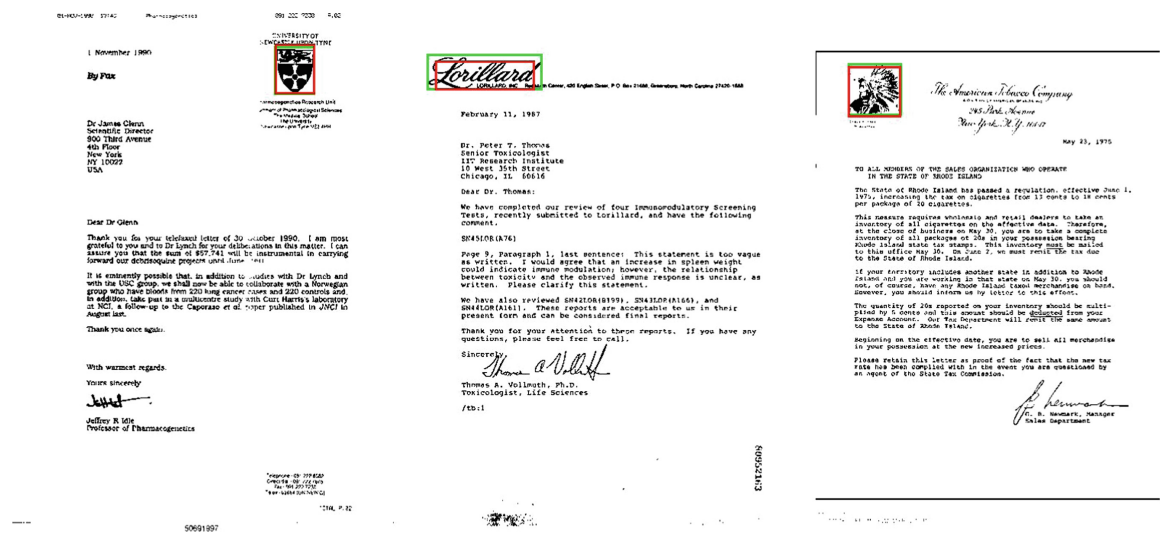

Fig. 4. Examples of qualitative evaluations on the Tobacco- 800 dataset: predicted bounding boxes (in red), and ground truth bounding boxes (in green) (Color figure online)
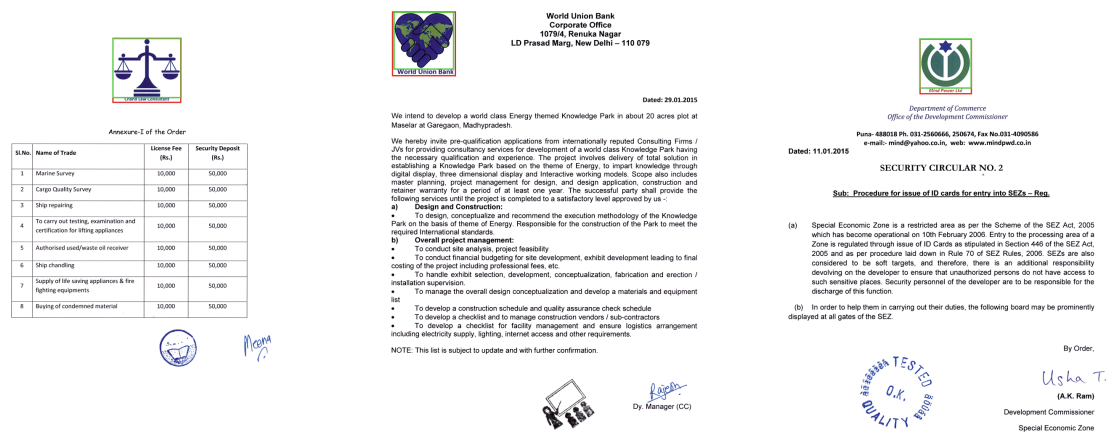

Fig. 5. Examples of qualitative evaluations on the SPODS dataset: predicted bounding boxes (in red), and ground truth bounding boxes (in green) (Color figure online)

We notice that the evaluation measures are higher than those obtained by the other methods. The considerable improvement in accuracy proofs the potential of the proposed method for detecting logos. Other advantages of our approach over state-of-the-art methods is that it is scalable for the logo scale variation and it does not require a-priori knowledge on processed images nor a training step with a huge amount of training data to have a reliable detection. Compared to block-based methods, this technique is simpler and more effective using only one suitable texture feature. According to the obtained results, we can affirm that the proposed approach is more effective than many other state-of-the-art logo detection methods. 
Table 1. Comparison of document logo detection performances

\begin{tabular}{l|l|l|l|l}
\hline $\begin{array}{l}\text { Document logo detection } \\
\text { techniques }\end{array}$ & Dataset & Images & Accuracy (\%) & Precision (\%) \\
\hline Veershetty and Hangarge [4] & Tobacco-800 & 200 & - & 87.80 \\
Naganjaneyulu et al. [5] & Tobacco-800 & 1151 & 91.47 & 98.10 \\
Li et al. [15] & Tobacco-800 & 1290 & 86.50 & 99.40 \\
Pham et al. [16] & Tobacco-800 & 426 & 90.05 & 92.98 \\
Dixit and Shirdhonkar [17] & Tobacco-800 & 1290 & 89.52 & - \\
Jain and Doermann [18] & Tobacco-800 & 435 & - & 87.00 \\
Le et al. [19] & Tobacco-800 & 1290 & 88.78 & 91.15 \\
Kumar and Ranjith [20] & Tobacco-800 & 500 & 91.30 & - \\
Wiggers et al. [21] & Tobacco-800 & 1290 & - & 72 \\
Alaei et al. [22] & Tobacco-800 & 1290 & 91.50 & 75.25 \\
Proposed approach & Tobacco-800 & 1290 & 96.86 & 98.93 \\
\hline
\end{tabular}

\section{Conclusion}

In this work, we present an accurate, effective, and efficient approach for document logo detection. The basic logo detection problem is the difficulty of accurately processing documents with a high degree of degradation and being unable to process correctly on unusual fonts. The traditional approaches to logo detection require a-priori information on processed images. Our solution employs the textural information to distinguish between logo and non-logo regions. We designed a complete detection pipeline containing a texture feature extraction and a fuzzy classification. Experiments are carried out on SPODS and Tobacco800 dataset. Our solution outperforms the methods in the state-of-the-art. However, there exist many unsolved challenges such as the generalization of this method to do logo recognition and other logical document components. We will attempt to address these issues in our future work.

\section{References}

1. Soon, F.C., Khaw, H.Y., Chuah, J.H., Kanesan, J.: Hyper-parameters optimisation of deep CNN architecture for vehicle logo recognition. IET Intel. Transp. Syst. 12, 939-946 (2018)

2. Tuzko, A., Herrmann, C., Manger, D., Beyerer, J.: Open set logo detection and retrieval. In: Proceedings of the 13th International Joint Conference on Computer Vision, Imaging and Computer Graphics Theory and Applications, pp. 284-292. SciTePress, Madeira (2018)

3. Alaei, A., Roy, P.P., Pal, U.: Logo and seal based administrative document image retrieval: a survey. Comput. Sci. Rev. 22, 47-63 (2016)

4. Veershetty, C., Hangarge, M.: Logo retrieval and document classification based on LBP features. In: Nagabhushan, P., Guru, D.S., Shekar, B.H., Kumar, Y.H.S. (eds.) 
Data Analytics and Learning. LNNS, vol. 43, pp. 131-141. Springer, Singapore (2019). https://doi.org/10.1007/978-981-13-2514-4_12

5. Naganjaneyulu, G.V.S.S.K.R., Sai Krishna, C., Narasimhadhan, A.V.: A novel method for logo detection based on curvelet transform using GLCM features. In: Chaudhuri, B.B., Kankanhalli, M.S., Raman, B. (eds.) Proceedings of 2nd International Conference on Computer Vision \& Image Processing. AISC, vol. 704, pp. 1-12. Springer, Singapore (2018). https://doi.org/10.1007/978-981-10-7898-9_1

6. Zaaboub, W., Dhiaf, Z.B.: Approach of texture signature determinationapplication to forest cover classification of high resolution satellite image. In: 6th International Conference of Soft Computing and Pattern Recognition (SoCPaR), pp. 325-330. IEEE (2014)

7. Krim, J., Indekeu, J.: Roughness exponents: a paradox resolved. Phys. Rev. E 48, 1576 (1993)

8. Turiel, A., Parga, N.: The multifractal structure of contrast changes in natural images: from sharp edges to textures. Neural Comput. 12, 763-793 (2000)

9. Gu, Q., Li, Z., Han, J.: Generalized fisher score for feature selection. arXiv preprint arXiv:1202.3725 (2012)

10. Miyamoto, S., Ichihashi, H., Honda, K., Ichihashi, H.: Algorithms for Fuzzy Clustering. Springer, Heidelberg (2008). https://doi.org/10.1007/978-3-540-78737-2

11. Soille, P.: Morphological Image Analysis: Principles and Applications. Springer, Heidelberg (2013). https://doi.org/10.1007/978-3-662-05088-0

12. Lewis, D., Agam, G., Argamon, S., Frieder, O., Grossman, D., Heard, J.: Building a test collection for complex document information processing. In: Proceedings of the 29th Annual International ACM SIGIR Conference on Research and Development in Information Retrieval, pp. 665-666. ACM (2006)

13. Nandedkar, A.V., Mukherjee, J., Sural, S.: SPODS: a dataset of color-official documents and detection of logo, stamp, and signature. ICVGIP 2016. LNCS, vol. 10481, pp. 219-230. Springer, Cham (2017). https://doi.org/10.1007/978-3-31968124-5_19

14. Alaei, A., Delalandre, M.: A complete logo detection/recognition system for document images. In: 2014 11th IAPR International Workshop on Document Analysis Systems (DAS), pp. 324-328. IEEE (2014)

15. Li, Z., Schulte-Austum, M., Neschen, M.: Fast logo detection and recognition in document images. In: 2010 International Conference on Pattern Recognition, pp. 2716-2719. IEEE (2010)

16. Pham, T.A., Delalandre, M., Barrat, S.: A contour-based method for logo detection. In: 2011 International Conference on Document Analysis and Recognition, pp. 718-722. IEEE (2011)

17. Dixit, U.D., Shirdhonkar, M., Automatic logo detection and extraction using singular value decomposition. In: International Conference on Communication and Signal Processing (ICCSP), pp. 0787-0790. IEEE (2016)

18. Jain, R., Doermann, D.: Logo retrieval in document images. In: 2012 10th IAPR International Workshop on Document Analysis Systems (DAS), pp. 135-139. IEEE (2012)

19. Le, V.P., Nayef, N., Visani, M., Ogier, J.M., De Tran, C.: Document retrieval based on logo spotting using key-point matching. In: 2014 22nd International Conference on Pattern Recognition (ICPR), pp. 3056-3061. IEEE (2014)

20. Sharath Kumar, Y.H., Ranjith, K.C.: An approach for logo detection and retrieval in documents. In: Santosh, K.C., Hangarge, M., Bevilacqua, V., Negi, A. (eds.) RTIP2R 2016. CCIS, vol. 709, pp. 49-58. Springer, Singapore (2017). https://doi. org/10.1007/978-981-10-4859-3_5 
21. Wiggers, K.L., Britto, A.S., Heutte, L., Koerich, A.L., Oliveira, L.E.S.: Document image retrieval using deep features. In: International Joint Conference on Neural Networks (IJCNN), pp. 1-8. IEEE (2018)

22. Alaei, A., Delalandre, M., Girard, N.: Logo detection using painting based representation and probability features. In: 12 th International Conference on Document Analysis and Recognition (ICDAR), pp. 1235-1239. IEEE (2013) 